\title{
Upregulation of vimentin and aberrant expression of E-cadherin/ $\beta$-catenin complex in oral squamous cell carcinomas: correlation with the clinicopathological features and patient outcome
}

\author{
Lai-Kui Liu $^{1,2}$, Xiao-Yun Jiang ${ }^{2,3}$, Xiang-Xiang Zhou ${ }^{2}$, Dong-Miao Wang, ${ }^{2,3}$ Xiao-Ling Song ${ }^{1}$ \\ and Hong-Bing Jiang ${ }^{2,3}$
}

${ }^{1}$ Department of Oral Pathology, College of Stomatology, Nanjing Medical University, Jiangsu, China; ${ }^{2}$ Department of Oral Pathology, Institute of Stomatology, Nanjing Medical University, Jiangsu, China and ${ }^{3}$ Department of Oral and Maxillofacial Surgery, College of Stomatology, Nanjing Medical University, Jiangsu, China

\begin{abstract}
Oral squamous cell carcinoma is a challenging oncology problem. A reliable biomarker for metastasis or high-risk prognosis in oral cancer patients remains undefined. Using quantitative immunohistochemistry, we examined the expression of vimentin, E-cadherin, and $\beta$-catenin in 83 oral squamous cell carcinoma patients, and the relationships between the expression of these markers and specific clinicopathological features were analysed. The high expression of vimentin was observed in 23 of $43(53 \%)$ tumours from patients who eventually developed a recurrent tumour and was associated with recurrence and death $(\boldsymbol{P}<0.001$ and $<0.001$, respectively). The decreased expression of E-cadherin was observed in 36 of $43(84 \%)$ tumours from patients who eventually developed a recurrent tumour and was also associated with recurrence and death $(P<0.001$ and $<0.001$, respectively). Although no correlation between $\beta$-catenin expression in whole-tumour sections and clinicopathological features was observed, decreased $\beta$-catenin expression at the tumour invasive front was closely associated with recurrence and death $(P=0.002$ and 0.002 , respectively). The expression of vimentin and that of E-cadherin were associated with survival and were independent prognostic factors in univariate and multivariate analyses. Our data show that the overexpression of vimentin was closely associated with recurrence and death in oral squamous cell carcinoma patients. The combination of the upregulation of vimentin and aberrant expression of $E$-cadherin/ $\beta$-catenin complexes at the tumour invasive front may provide a useful prognostic marker in oral squamous cell carcinoma.

Modern Pathology (2010) 23, 213-224; doi:10.1038/modpathol.2009.160; published online 13 November 2009
\end{abstract}

Keywords: vimentin; oral squamous cell carcinoma; immunohistochemistry

Oral cancer is the sixth most frequently occurring cancer worldwide, accounting for $3-5 \%$ of all malignancies in both sexes. ${ }^{1,2}$ Over $90 \%$ of all oral

Correspondence: Dr L-K Liu, Department of Oral Pathology, College of Stomatology, Nanjing Medical University, 136\# Hanzhong Road, Nanjing, Jiangsu 210029, China.

E-mail: laikuiliu@sina.com

or Dr H-B Jiang, Department of Oral and Maxillofacial Surgery, College of Stomatology, Nanjing Medical University, 136\# Hanzhong Road, Nanjing, Jiangsu 210029, China.

E-mail: jhbcd@sina.com

Received 29 June 2009; revised 8 October 2009; accepted 11 October 2009; published online 13 November 2009 carcinomas are classified as oral squamous cell carcinoma, which remains a challenging oncology problem. ${ }^{3}$ Although early-stage oral squamous cell carcinoma can be treated or cured, the prognosis for advanced oral squamous cell carcinoma (stage III and IV) is poor. The treatment of oral squamous cell carcinoma is usually based on surgery or radiation, with or without concomitant chemotherapy. Despite these advanced therapeutic strategies, the 5-year survival rate of oral squamous cell carcinoma $(\sim 50 \%)$ has not increased over the past four decades. $^{3-5}$ Local or regional relapse and cervical lymph node metastasis are the most prevalent 
causes of death in these patients, but these processes are poorly understood. If a reliable biomarker for metastasis and high-risk prognoses in oral squamous cell carcinoma patients could be identified, it would aid in definitive treatment planning, provide clues for selection of a treatment strategy, and could improve survival. However, to date, few reports have examined markers to predict metastasis, ${ }^{6,7}$ and the molecular mechanisms mediating invasion and metastasis of oral squamous cell carcinoma remain undefined. Therefore, there is a need to better understand the molecular cause of oral squamous cell carcinoma progression to develop new treatment options.

Studies of epithelial malignancies have consistently shown that the transition from an epithelial cell to a mesenchymal cell, as characterised by the loss of E-cadherin expression, the aberrant expression of E-cadherin/ $\beta$-catenin complexes, or the gain of vimentin expression, is correlated with the conversion of early-stage tumours to invasive malignancies. $^{8-10}$ This process is similar to the epithelialmesenchymal transition that has been implicated in tissue remodelling, organ development, wound healing, and cancer progression. ${ }^{9-12}$ The epithelial-mesenchymal transition is a complex process that governs morphogenesis in multicellular organisms and can lead to a spectrum of epithelial cellular changes, including loss of polarity and adhesion, increased motility, and the acquisition of a mesenchymal phenotype. ${ }^{9-12}$ Several studies of epithelial malignancies have shown that E-cadherin or $\beta$-catenin can have a transcriptional and regulatory role in invasion and metastasis and is associated with a poor outcome. ${ }^{13-23}$

E-cadherin is a $120 \mathrm{kDa}$ calcium-dependent transmembrane glycoprotein encoded by the $C D H 1$ gene located on chromosome 16q21, and it is expressed in most epithelial cells. ${ }^{18}$ E-cadherin has a major role in establishing cell polarity and in maintaining normal tissue architecture. The intracellular domain of E-cadherin is linked to the actin cytoskeleton through its interaction with its cytoplasmic-binding partners, the catenins $\left(\alpha-, \beta\right.$-, and $\gamma$-catenin). ${ }^{24}$ The E-cadherin/ $\beta$-catenin complex, therefore, functions as a component of adherent cell-cell junctions that promote cell adhesion. ${ }^{13,24}$ The accumulation of free cytoplasmic $\beta$-catenin and/or its translocation to the nucleus (where it acts as a transcriptional activator through its binding with the members of the TCF/LEF1 family) has been associated with various physiological and pathological processes, including tumour progression..$^{25,26}$

In addition to the aberrant expression of Ecadherin/ $\beta$-catenin complexes, the de novo expression of vimentin has been frequently associated with the metastatic conversion of epithelial cells and tumour invasion. Vimentin is a type III intermediate filament protein normally found in mesenchymal cells. ${ }^{27}$ Numerous data have shown that vimentin can be expressed in migratory epithelial cells that are involved in embryogenesis and organogenesis, wound healing, or tumour invasion. ${ }^{28-31}$ Gilles et $a 1^{29}$ have shown that the vimentin promoter is a target of the $\beta$-catenin/TCF pathway, which suggests that this functional regulation of epithelial cells is involved in invasion and/or migration. ${ }^{32}$

Currently, it is thought that the invasive front of tumours is the region containing the most useful prognostic information because, presumably, the most invasive cells are located there. ${ }^{33,34}$ The histological features of oral squamous cell carcinoma may vary widely within the same tumour from the central area to the invasive area. The tumour grade at the invasive front (the histological grade at the deep invasive front) has been shown to have a high prognostic value for oral squamous cell carcinoma. ${ }^{6,33,34}$ Therefore, it is very important to evaluate the tumour invasive front and identify molecular biomarkers that can help clinicians decide the best treatment strategy. In this study, we investigated the expression of vimentin and Ecadherin/ $\beta$-catenin expression in oral squamous cell carcinomas and examined the potential role of the epithelial-mesenchymal transition in invasiveness through its association with clinicopathological features and patient outcome.

\section{Materials and methods}

\section{Patients and Tissue Specimens}

A total of 83 primary oral squamous cell carcinoma biopsy specimens from patients diagnosed from 1994 to 2004 were obtained from the files of the Department of Oral Pathology and the Department of Oral Maxillofacial Surgery, College of Stomatology, Nanjing Medical University. Detailed clinicopathological information was obtained from patient records. Two pathologists (Drs XL Song and SX Wang from the Department of Oral Pathology, College of Stomatology, Nanjing Medical University) evaluated haematoxylin and eosin-stained tissue sections to confirm or correct the previous histological diagnoses according to the revised criteria suggested by the World Health Organization (2005). All the patients had been surgically treated with a wide excision of the primary tumour with a simultaneous classical radical neck dissection or an elective dissection of the regional lymph nodes. The primary treatments were given at our centre. None of the patients had received any form of tumour-specific therapy before the total surgical excision of the lesion. This study protocol was approved by the Ethics Committee (Institutional Review Board) of the Nanjing Medical University.

\section{Histopathological Evaluation}

The histological characteristics of oral squamous cell carcinoma were classified into well-, moder- 
ately, and poorly differentiated groups (G1-G3) according to the criteria proposed by the World Health Organization.

The clinical staging and TNM classification were determined according to the International Union Against Cancer tumour classification for each patient: the $\mathrm{T}$ classification was into the $\mathrm{T} 1, \mathrm{~T} 2$, $\mathrm{T} 3$, and $\mathrm{T} 4$ categories and the $\mathrm{N}$ classification was into lymph node-negative (No) and lymph nodepositive $(\mathrm{N})$ categories. The stage grouping was divided into the stage I, I, III, and IV categories.

\section{Pattern of Invasion}

The pattern of tumour invasion was examined at the normal tissue-tumour interface. The pattern of invasion was classified into four types according to Bryne's classification. ${ }^{6,35,36}$ Pattern of invasion type one represents tumour invasion in a broad pushing manner with a well-delineated infiltrating border. Pattern of invasion type two represents tumour invasion with broad pushing 'fingers' or separate large tumour islands, with a stellate appearance. Pattern of invasion type three represents invasive tumour islands greater than 15 cells per island. Pattern of invasion type four represents invasive islands of tumour smaller than 15 cells per island, including cord-like and single-cell invasions.

\section{Immunohistochemistry}

Specimens from all cases were fixed in a $10 \%$ formaldehyde solution and embedded in paraffin. Briefly, tissue sections ( $4-5 \mu \mathrm{m})$ from representative paraffin blocks were deparaffinised in xylene and rehydrated through graded alcohols. Endogenous peroxidases were blocked using 3\% hydrogen peroxide. For antigen retrieval, the sections were processed by conventional microwave heating in $0.01 \mathrm{M}$ sodium citrate retrieval buffer ( $\mathrm{pH}$ 6.0) for $20 \mathrm{~min}$. The sections were then incubated with primary antibody for $60 \mathrm{~min}$ at room temperature and subsequently incubated with goat anti-mouse EnVision (Dako, Carpinteria, CA, USA) for E-cadherin (mouse monoclonal antibody, ZM-0092, Zymed Laboratories, San Diego, CA, USA) or $\beta$-catenin (mouse monoclonal antibody, sc-7963, Santa Cruz Biotechnology), or goat anti-rabbit EnVision (Dako) for vimentin (rabbit monoclonal antibody, ZM-0511, Southern California, Zymed Laboratories) for $30 \mathrm{~min}$ at room temperature. The sections were then washed three times with phosphate-buffered saline ( $\mathrm{pH}$ 7.2) for $2 \mathrm{~min}$. The reaction product was developed with 3,3'-diaminobenzidine and counterstained with haematoxylin. Immunoreactivity in the tissue was judged independently by two pathologists who were blinded to the clinical data and other immunohistochemical results. Normal oral mucosal tissues were used as a positive control. Negative controls were included in each slide run (omission of primary and secondary antibodies), and all controls gave appropriate results.

\section{Evaluation of Immunoreactivity}

Immunoreactivity was semiquantitatively evaluated on the basis of staining intensity and distribution using the immunoreactive score: ${ }^{37,38}$ Immunoreactive score $=$ intensity score $\times$ proportion score. The intensity score was defined as 0 , negative; 1 , weak; 2 , moderate; or 3, strong, and the proportion score was defined as 0 , negative; $1,<10 \% ; 2,11-50 \%$; $3,51-80 \%$; or $4,>80 \%$ positive cells. The total score ranged from 0 to 12 . The immunoreactivity was divided into three groups on the basis of the final score: negative immunoreactivity was defined as a total score of 0 , low immunoreactivity was defined as a total score of 1-4, and high immunoreactivity was defined as a total score $>4$. The immunostaining of the tumour invasive front was evaluated using the same method. The stained tumour tissues were scored, although blinded to the clinical patient data.

\section{Follow-Up and Statistical Analysis}

To determine the prognostic factor, the outcome of the 83 patients was determined by reviewing their medical charts. The follow-up period ranged from 2 to 170 months (average: 50.1 months; median: $\mathbf{5 1 . 0}$ months). In total, 60 patients had at least a 2-year follow-up after treatment. The end point in the analysis was carcinoma-related death. The overall and disease-free survival rate was estimated using the Kaplan-Meier method and compared by the log-rank test. A prognostic analysis was carried out with univariate and multivariate Cox regressions models. The correlation between the clinicopathological parameters and vimentin, E-cadherin, and $\beta$-catenin expression was analysed using the $\chi^{2}$-test, Fisher's exact test, or the Kruskal-Wallis $H$-test. The associations between vimentin, E-cadherin, and $\beta$-catenin expression were analysed using the $\chi^{2}$-tests for a linear trend. The data were analysed using SPSS statistical software (for Windows, version 16.0). A $P$-value $<0.05$ was considered statistically significant.

\section{Results}

\section{Clinicopathological Characteristics}

A total of 83 cases of primary oral squamous cell carcinoma were analysed, 46 men (55\%) and 37 women $(45 \%)$ (mean age 58.0 years, range 26-79 years). The main clinical characteristics of the patients analysed in this study are detailed in Table 1. 
Table 1 Relationship between vimentin, E-cadherin, and $\beta$-catenin expression levels of tumors and clinical variables

\begin{tabular}{|c|c|c|c|c|c|c|c|c|c|c|c|c|c|}
\hline \multirow[t]{2}{*}{ Variable } & \multirow[t]{2}{*}{ No. } & \multicolumn{4}{|c|}{ Vimentin } & \multicolumn{4}{|c|}{ E-cadherin } & \multicolumn{4}{|c|}{$\beta$-Catenin } \\
\hline & & $\mathrm{N}$ & $L$ & $H$ & $\mathrm{P}$-value & $N$ & $L$ & $H$ & $\mathrm{P}$-value & $\mathrm{N}$ & $L$ & $H$ & $\mathrm{P}$-value \\
\hline Sex & & & & & 0.113 & & & & 0.929 & & & & 0.109 \\
\hline Male & 46 & 18 & 12 & 16 & & 19 & 9 & 18 & & 4 & 11 & 31 & \\
\hline Female & 37 & 8 & 17 & 12 & & 16 & 8 & 13 & & 9 & 5 & 23 & \\
\hline Age (years) & & & & & 0.523 & & & & 0.753 & & & & 0.757 \\
\hline$\leq 50$ & 25 & 10 & 8 & 7 & & 12 & 5 & 8 & & 4 & 6 & 15 & \\
\hline$>50$ & 58 & 16 & 21 & 21 & & 23 & 12 & 23 & & 9 & 10 & 39 & \\
\hline Tumor location & & & & & 0.786 & & & & 0.607 & & & & 0.928 \\
\hline Tongue & 30 & 12 & 9 & 9 & & 14 & 5 & 11 & & 7 & 5 & 18 & \\
\hline Buccal mucosa & 23 & 5 & 8 & 10 & & 8 & 4 & 11 & & 2 & 4 & 17 & \\
\hline Gingiva & 11 & 3 & 4 & 4 & & 5 & 1 & 5 & & 2 & 3 & 6 & \\
\hline Palate & 8 & 1 & 5 & 2 & & 3 & 4 & 1 & & 1 & 2 & 5 & \\
\hline Lip & 3 & 1 & 1 & 1 & & 2 & 1 & 0 & & 1 & 0 & 2 & \\
\hline Floor of the mouth & 2 & 1 & 1 & 0 & & 0 & 1 & 1 & & 0 & 1 & 1 & \\
\hline Retromolar pad & 2 & 1 & 1 & 0 & & 1 & 0 & 1 & & 0 & 0 & 2 & \\
\hline Others & 4 & 2 & 0 & 2 & & 2 & 1 & 1 & & 0 & 1 & 3 & \\
\hline Histological differentiation & & & & & 0.568 & & & & 0.834 & & & & 0.250 \\
\hline Well & 49 & 16 & 19 & 14 & & 21 & 9 & 19 & & 8 & 12 & 29 & \\
\hline Moderate & 30 & 9 & 8 & 13 & & 12 & 7 & 11 & & 5 & 4 & 21 & \\
\hline Poor & 4 & 1 & 2 & 1 & & 2 & 1 & 1 & & 0 & 0 & 4 & \\
\hline Pattern of invasion & & & & & 0.447 & & & & 0.404 & & & & 0.932 \\
\hline 1 & 15 & 6 & 5 & 4 & & 5 & 2 & 8 & & 4 & 2 & 9 & \\
\hline 2 & 36 & 12 & 13 & 11 & & 17 & 7 & 12 & & 3 & 8 & 25 & \\
\hline 3 & 20 & 4 & 9 & 7 & & 6 & 5 & 9 & & 3 & 3 & 14 & \\
\hline 4 & 12 & 4 & 2 & 6 & & 7 & 3 & 2 & & 3 & 3 & 6 & \\
\hline Tumor size & & & & & 0.255 & & & & 0.288 & & & & 0.723 \\
\hline $\mathrm{T} 1$ & 20 & 7 & 4 & 9 & & 12 & 3 & 5 & & 4 & 5 & 11 & \\
\hline $\mathrm{T} 2$ & 32 & 6 & 15 & 11 & & 10 & 10 & 12 & & 5 & 5 & 22 & \\
\hline $\mathrm{T} 3$ & 9 & 3 & 2 & 4 & & 3 & 3 & 3 & & 0 & 3 & 6 & \\
\hline $\mathrm{T} 4$ & 22 & 10 & 8 & 4 & & 10 & 1 & 11 & & 4 & 3 & 15 & \\
\hline Clinical stage & & & & & 0.430 & & & & 0.459 & & & & 0.726 \\
\hline I & 19 & 7 & 4 & 8 & & 12 & 2 & 5 & & 3 & 5 & 11 & \\
\hline II & 30 & 6 & 14 & 10 & & 8 & 10 & 12 & & 4 & 5 & 21 & \\
\hline III & 12 & 3 & 3 & 6 & & 5 & 4 & 3 & & 2 & 3 & 7 & \\
\hline IV & 22 & 10 & 8 & 4 & & 10 & 1 & 11 & & 4 & 3 & 15 & \\
\hline Nodal metastasis & & & & & 0.134 & & & & 0.586 & & & & 0.828 \\
\hline No & 51 & 18 & 20 & 13 & & 21 & 9 & 21 & & 7 & 10 & 34 & \\
\hline $\mathrm{N}(+)$ & 32 & 8 & 9 & 15 & & 14 & 8 & 10 & & 6 & 6 & 20 & \\
\hline Recurrence & & & & & $<0.001$ & & & & $<\mathbf{0 . 0 0 1}$ & & & & 0.056 \\
\hline No & 40 & 17 & 18 & 5 & & 11 & 5 & 24 & & 5 & 4 & 31 & \\
\hline Yes & 43 & 9 & 11 & 23 & & 24 & 12 & 7 & & 8 & 12 & 23 & \\
\hline Follow-up & & & & & $<0.001$ & & & & $<\mathbf{0 . 0 0 1}$ & & & & 0.091 \\
\hline Live without recurrence & 40 & 17 & 18 & 5 & & 11 & 5 & 24 & & 5 & 4 & 31 & \\
\hline Died of recurrence & 41 & 8 & 10 & 23 & & 24 & 12 & 5 & & 8 & 12 & 21 & \\
\hline Live with recurrence & 2 & 1 & 1 & 0 & & 0 & 0 & 2 & & 0 & 0 & 2 & \\
\hline
\end{tabular}

No., number of patients; N, negative; L low expression; H, high expression; N0, no nodal metastasis; N (+), nodal metastasis. Bold values signify $P$-value $<0.05$.

\section{Immunohistochemical Analysis}

Expression of vimentin, E-cadherin, and $\beta$-catenin in oral squamous cell carcinoma patients

The immunohistochemical staining results for vimentin, E-cadherin, and $\beta$-catenin are presented in Table 1.
Vimentin: Vimentin was detected in the cytoplasm of the connective tissue mesenchymal cells of the normal oral mucosal tissue, but not in the normal squamous epithelium (Figure 1a). Of the 83 tumours, 57 (69\%) exhibited weak or strong cytoplasmic immunoreactivity for vimentin (immunoreactive score $=$ low expression, 29 cases; 

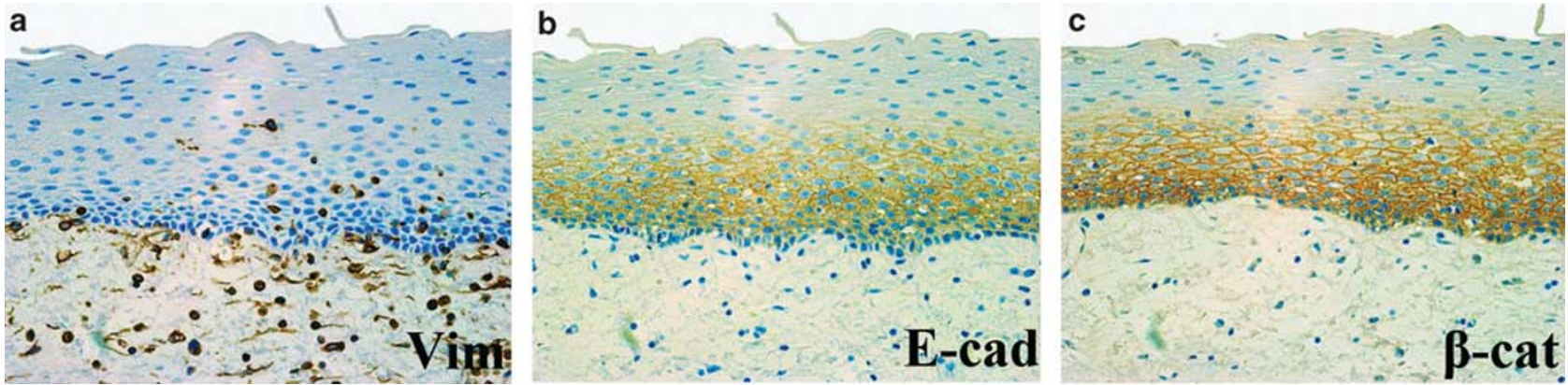

Figure 1 Vimentin, E-cadherin, and $\beta$-catenin expression was examined by immunohistochemistry. Negative staining of vimentin (a), strong membranous staining of E-cadherin (b), and membranous or cytoplasmic staining of $\beta$-catenin (c) were found in normal oral epithelia. $(\mathbf{a}-\mathbf{c}, \times 400)$.
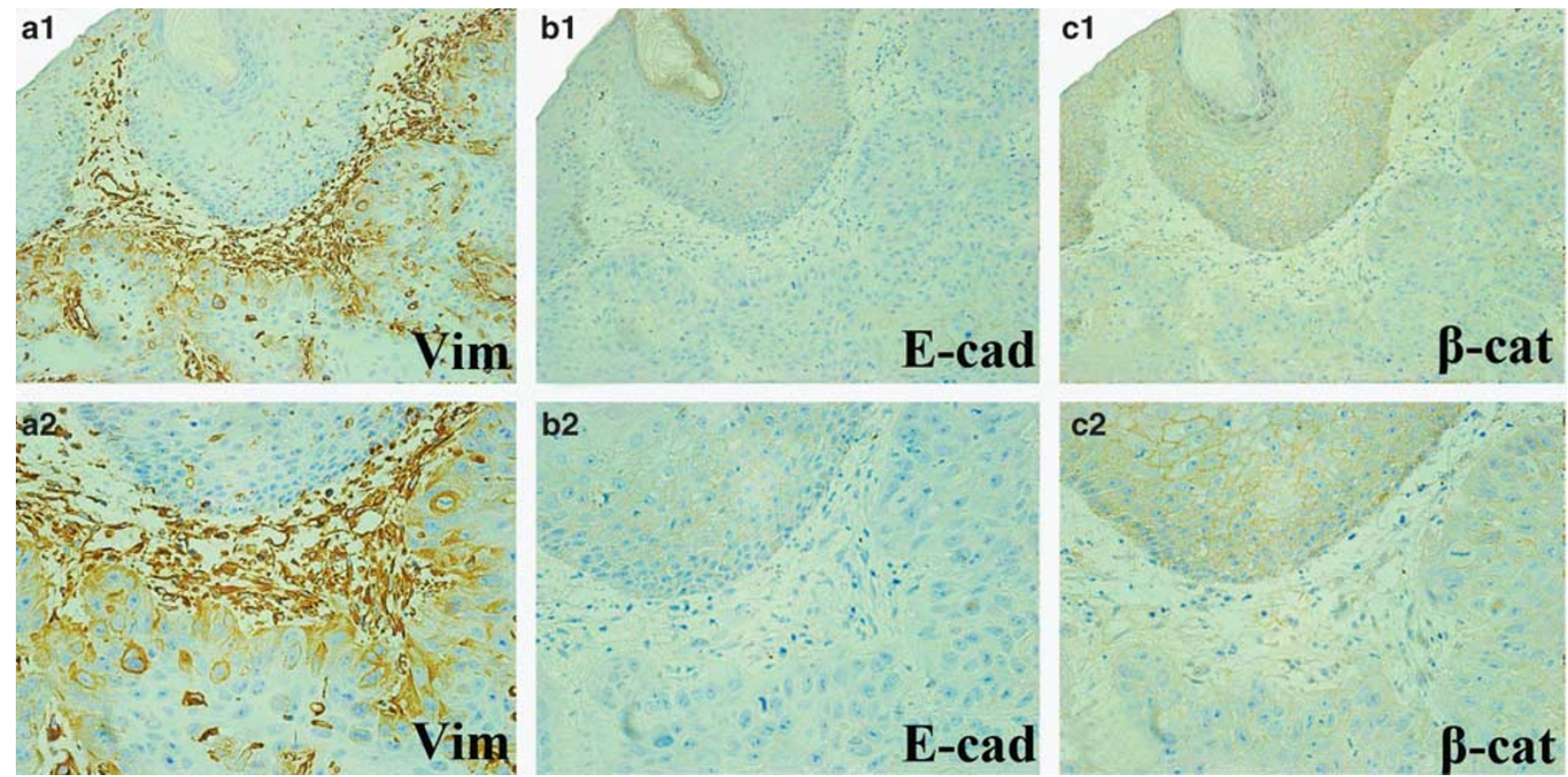

Figure 2 Vimentin $(\mathbf{a} 1, \mathbf{a} 2)$, E-cadherin $(\mathbf{b} 1, \mathbf{b} 2)$, and $\beta$-catenin $(\mathbf{c} 1, \mathbf{c} 2)$ expression was examined by immunohistochemistry. In oral squamous cell carcinoma patients, the absence or reduced expression of E-cadherin (b1, b2) or $\beta$-catenin (c1, c2) and increased cytoplasmic staining of vimentin $(\mathbf{a} 1, \mathbf{a} 2)$ was observed in cancerous tissue. $(\mathbf{a} 1-\mathbf{c 1}, \times 200 ; \mathbf{a} 2-\mathbf{c} 2, \times 400)$.

immunoreactive score $=$ high expression, 28 cases). The remaining $26(31 \%)$ were negative (immunoreactive score $=0$ ) (Figures 2a1, a2). Increased cytoplasmic staining of vimentin was detectable in the majority of finger-like invasive fronts of tumours (Figures 3a1, a2). The staining of vimentin showed no significant differences between the well-, moderately, and poorly differentiated tumours.

E-cadherin: In normal oral mucosa, distinct membranous expression of E-cadherin was observed mainly on the cell membrane of the basal layer of the spinosum layers (Figure 1b). In cancerous tissue from oral squamous cell carcinoma patients, 48 (58\%) of 83 tumours exhibited membranous or cytoplasmic E-cadherin expression (immunoreactive score $=$ low expression, 17 cases; immunoreactive score $=$ high expression, 31 cases) (Figures 2b1, b2). The remaining
$35(42 \%)$ were negative (immunoreactive score $=0$ ). Especially at the invasive front of the tumour, the E-cadherin expression was frequently lost (54\%) (immunoreactive score $=0,45$ cases; immunoreactive score $=$ low expression, 16 cases; immunoreactive score $=$ high expression, 22 cases) (Figures 3b1, b2).

$\beta$-Catenin: In normal oral mucosa, membranous or cytoplasmic expression of $\beta$-catenin was observed in the suprabasal to the basal cell layer (Figure 1c). In cancerous tissue, the majority of the 83 tumour samples (70 of $83,84 \%$ ) exhibited membranous or cytoplasmic expression of $\beta$-catenin (immunoreactive score $=$ low expression, 16 cases; immunoreactive score $=$ high expression, 54 cases) (Figures 2c1, c2). The remaining $13(16 \%)$ were negative (immunoreactive score $=0$ ). A shift in the $\beta$-catenin expression (22 of 83, 27\%) was observed 

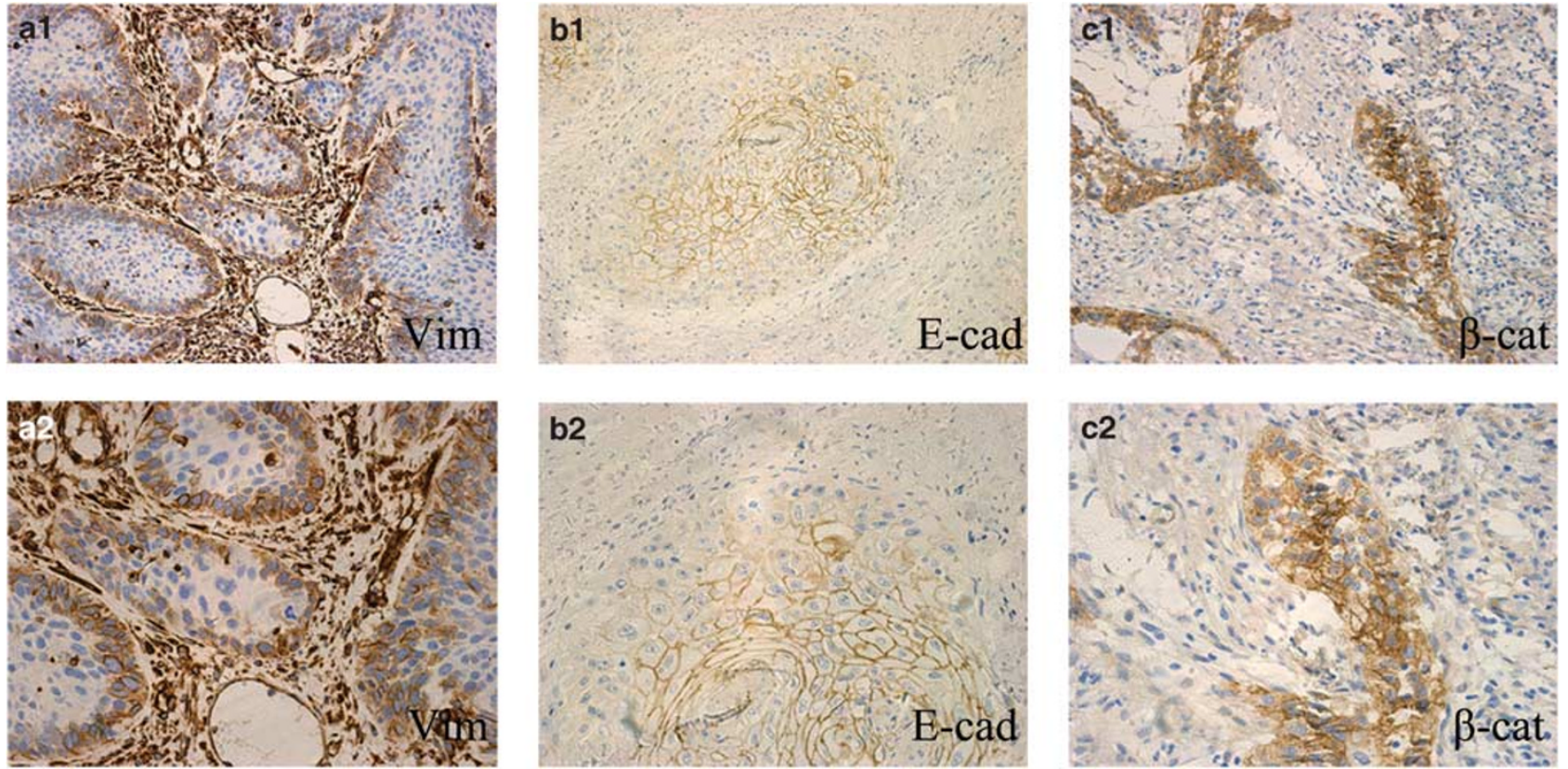

Figure 3 Immunohistochemical expression of vimentin $(\mathbf{a} 1, \mathbf{a} 2)$, E-cadherin $(\mathbf{b} 1, \mathbf{b} 2)$, and $\beta$-catenin $(\mathbf{c} 1$, $\mathbf{c} 2)$ at the invasive front of oral squamous cell carcinoma. Strong cytoplasmic staining of vimentin $(\mathbf{a} 1, \mathbf{a} 2)$ was found at the tumour invasive front, whereas there was no staining or low expression of E-cadherin $(\mathbf{b} 1, \mathbf{b} 2)$. A shift of the $\beta$-catenin expression pattern (c1, $\mathbf{c} 2)$ was observed from the membrane or cytoplasm toward the nucleus and/or cytoplasm at the invasive front of oral squamous cell carcinoma. (a1-c1, $\times 200$; a2-c2, $\times 400)$.

from membranous or cytoplasmic expression toward nuclear and/or cytoplasmic expression (immunoreactive score $=0,18$ cases; immunoreactive score $=$ low expression, 18 cases; immunoreactive score $=$ high expression, 47 cases), particularly at the invasive front of the tumour (Figures 3c1, c2).

Association of the clinicopathological variables with the expression levels of vimentin, E-cadherin, and $\beta$-catenin

The relationship between the clinicopathological features and the expression levels of vimentin, E-cadherin, and $\beta$-catenin is shown in Table 1 . There were no statistically significant differences between the expression levels of vimentin, E-cadherin, or $\beta$-catenin and sex, age, tumour location, histological differentiation, pattern of invasion, tumour size, clinical stage, or the presence/absence of regional lymph node spread. Similarly, there were no statistically significant differences between $\beta$-catenin expression and the absence/presence of tumour recurrence or death from recurrence. However, the increased expression of vimentin was observed in $53 \%$ (23 out of 43 ) of tumours from patients who eventually developed a recurrent tumour and $56 \%$ (23 out of 41) of tumours from patients who died of recurrence, whereas $13 \%$ (5 out of 40 ) of tumours from patients without a history of tumour recurrence expressed vimentin. There was a significant increase in vimentin expression in tumours from patients who eventually developed a recurrent tumour or in tumours from patients who died of recurrence, compared with tumours from patients without a history of tumour recurrence $(P<0.001$ and $<0.001$, respectively).

The absence or reduced expression of E-cadherin was observed in $84 \%$ (36 out of 43 ) of tumours from patients who eventually developed a recurrent tumour and $88 \%$ (36 out of 41) of tumours from patients who died of recurrence, whereas $40 \%$ (16 out of 40) of tumours from patients without a history of tumour recurrence lacked the expression of E-cadherin. There was a significant decrease in E-cadherin expression in tumours from patients who eventually developed a recurrent tumour or in tumours from patients who died of recurrence, compared with tumours from patients without a history of tumour recurrence $(P<0.001$ and $<0.001$, respectively).

The relationship between the vimentin, E-cadherin, and $\beta$-catenin expression at the tumour invasive front and the clinical variables is shown in Table 2. There were no statistically significant differences between the expression levels of vimentin, E-cadherin, and $\beta$-catenin at the tumour invasive front and sex, age, tumour location, histological differentiation, pattern of invasion, tumour size, clinical stage, or lymph node metastasis. However, the absence or reduced expression of $\beta$-catenin was observed in $60 \%$ (26 out of 43) of tumours from patients who eventually developed a recurrent tumour and 63\% (26 out of 41) of tumours from patients who died of recurrence, whereas $25 \%$ (10 out of 40 ) of tumours from patients without a history of tumour recurrence lacked $\beta$-catenin expression. There was a significant decrease in $\beta$-catenin expression at the tumour invasive front 
Table 2 Relationship between vimentin, E-cadherin, and $\beta$-catenin expression levels of the tumors invasive front and clinical variables

\begin{tabular}{|c|c|c|c|c|c|c|c|c|c|c|c|c|c|}
\hline \multirow[t]{2}{*}{ Variable } & \multirow[t]{2}{*}{ No. } & \multicolumn{4}{|c|}{ Vimentin } & \multicolumn{4}{|c|}{ E-cadherin } & \multicolumn{4}{|c|}{$\beta$-Catenin } \\
\hline & & $N$ & $L$ & $H$ & $\mathrm{P}$-value & $\mathrm{N}$ & $L$ & $H$ & $\mathrm{P}$-value & $N$ & $L$ & $H$ & $\mathrm{P}$-value \\
\hline Sex & & & & & 0.231 & & & & 0.624 & & & & 0.091 \\
\hline Male & 46 & 18 & 10 & 18 & & 23 & 9 & 14 & & 6 & 12 & 28 & \\
\hline Female & 37 & 8 & 10 & 19 & & 22 & 7 & 8 & & 12 & 6 & 19 & \\
\hline Age (years) & & & & & 0.484 & & & & 0.777 & & & & 0.313 \\
\hline$\leq 50$ or younger & 25 & 10 & 6 & 9 & & 15 & 4 & 6 & & 7 & 7 & 11 & \\
\hline$>50$ & 58 & 16 & 14 & 28 & & 30 & 12 & 16 & & 11 & 11 & 36 & \\
\hline Tumor location & & & & & 0.559 & & & & 0.755 & & & & 0.882 \\
\hline Tongue & 30 & 12 & 7 & 11 & & 17 & 4 & 9 & & 8 & 8 & 14 & \\
\hline Buccal mucosa & 23 & 5 & 5 & 13 & & 11 & 5 & 7 & & 3 & 6 & 14 & \\
\hline Gingiva & 11 & 3 & 2 & 6 & & 6 & 3 & 2 & & 3 & 2 & 6 & \\
\hline Palate & 8 & 1 & 4 & 3 & & 4 & 3 & 1 & & 2 & 1 & 5 & \\
\hline Lip & 3 & 1 & 0 & 2 & & 3 & 0 & 0 & & 2 & 0 & 1 & \\
\hline Floor of the mouth & 2 & 1 & 1 & 0 & & 0 & 1 & 1 & & 0 & 0 & 2 & \\
\hline Retromolar pad & 2 & 1 & 1 & 0 & & 1 & 0 & 1 & & 0 & 0 & 2 & \\
\hline Others & 4 & 2 & 0 & 2 & & 3 & 0 & 1 & & 0 & 1 & 3 & \\
\hline Histological differentiation & & & & & 0.904 & & & & 0.830 & & & & 0.160 \\
\hline Well & 49 & 16 & 11 & 22 & & 28 & 8 & 13 & & 12 & 13 & 24 & \\
\hline Moderate & 30 & 9 & 8 & 13 & & 14 & 8 & 8 & & 6 & 5 & 19 & \\
\hline Poor & 4 & 1 & 1 & 2 & & 3 & 0 & 1 & & 0 & 0 & 4 & \\
\hline Pattern of invasion & & & & & 0.620 & & & & 0.247 & & & & 0.397 \\
\hline 1 & 15 & 6 & 2 & 7 & & 6 & 2 & 7 & & 5 & 1 & 9 & \\
\hline 2 & 36 & 12 & 9 & 15 & & 21 & 7 & 8 & & 5 & 9 & 22 & \\
\hline 3 & 20 & 4 & 7 & 9 & & 9 & 5 & 6 & & 4 & 4 & 12 & \\
\hline 4 & 12 & 4 & 2 & 6 & & 9 & 2 & 1 & & 4 & 4 & 4 & \\
\hline Tumor size & & & & & 0.259 & & & & 0.234 & & & & 0.695 \\
\hline $\mathrm{T} 1$ & 20 & 7 & 2 & 11 & & 13 & 4 & 3 & & 6 & 4 & 10 & \\
\hline $\mathrm{T} 2$ & 32 & 6 & 11 & 15 & & 16 & 8 & 8 & & 6 & 8 & 18 & \\
\hline $\mathrm{T} 3$ & 9 & 3 & 1 & 5 & & 4 & 2 & 3 & & 2 & 1 & 6 & \\
\hline $\mathrm{T} 4$ & 22 & 10 & 6 & 6 & & 12 & 2 & 8 & & 4 & 5 & 13 & \\
\hline Clinical stage & & & & & 0.481 & & & & 0.371 & & & & 0.966 \\
\hline I & 19 & 7 & 2 & 10 & & 13 & 3 & 3 & & 5 & 4 & 10 & \\
\hline II & 30 & 6 & 11 & 13 & & 14 & 8 & 8 & & 5 & 7 & 18 & \\
\hline III & 12 & 3 & 1 & 8 & & 6 & 3 & 3 & & 4 & 2 & 6 & \\
\hline IV & 22 & 10 & 6 & 6 & & 12 & 2 & 8 & & 4 & 5 & 13 & \\
\hline Nodal metastasis & & & & & 0.092 & & & & 0.349 & & & & 0.828 \\
\hline No & 51 & 18 & 15 & 18 & & 27 & 8 & 16 & & 10 & 11 & 30 & \\
\hline $\mathrm{N}(+)$ & 32 & 8 & 5 & 19 & & 18 & 8 & 6 & & 8 & 7 & 17 & \\
\hline Recurrence & & & & & 0.001 & & & & $<\mathbf{0 . 0 0 1}$ & & & & 0.002 \\
\hline No & 40 & 17 & 14 & 9 & & 13 & 8 & 19 & & 7 & 3 & 30 & \\
\hline Yes & 43 & 9 & 6 & 28 & & 32 & 8 & 3 & & 11 & 15 & 17 & \\
\hline Follow-up & & & & & $<\mathbf{0 . 0 0 1}$ & & & & $<\mathbf{0 . 0 0 1}$ & & & & 0.002 \\
\hline Live without recurrence & 40 & 17 & 14 & 9 & & 13 & 8 & 19 & & 7 & 3 & 30 & \\
\hline Died of recurrence & 41 & 8 & 5 & 28 & & 32 & 8 & 1 & & 11 & 15 & 15 & \\
\hline Live with recurrence & 2 & 1 & 1 & 0 & & 0 & 0 & 2 & & 0 & 0 & 2 & \\
\hline
\end{tabular}

No., number of patients; N, negative; L, = low expression; H, high expression; N0, no nodal metastasis; N (+), nodal metastasis.

Bold values signify $P$-value $<0.05$.

in tumours from patients who eventually developed a recurrent tumour or tumours from patients who died of recurrence, compared with tumours from patients without a history of tumour recurrence $(P=0.002$ and 0.002 , respectively). There was a statistically significant increase in vimentin expression at the tumour invasive front in tumours from patients who eventually developed a recurrent tumour or tumours from patients who died of recurrence, compared with tumours from patients without a history of tumour recurrence $(P<0.001$ and $<0.001$, respectively). Similarly, there was also a statistically significant decrease in E-cadherin expression at the tumour invasive front in tumours from patients who eventually developed a recurrent tumour or tumours from patients who died of recurrence, compared with 
Table 3 Correlation between immunostaining of vimentin and E-cadherin, and $\beta$-catenin

\begin{tabular}{|c|c|c|c|c|c|c|c|c|}
\hline \multirow[t]{2}{*}{ Variable } & \multirow[t]{2}{*}{ Number } & \multicolumn{4}{|c|}{ Vimentin } & \multicolumn{3}{|c|}{$\beta$-Catenin } \\
\hline & & $N$ & $L$ & $H$ & P-value & $N$ & $L H$ & P-value \\
\hline E-cadherin & & & & & 0.035 & & & 0.001 \\
\hline Negative & 35 & 11 & 7 & 17 & & 10 & 817 & \\
\hline Low expression & 17 & 3 & 7 & 7 & & & 79 & \\
\hline High expression & 31 & 12 & 15 & 4 & & & 128 & \\
\hline$\beta$-Catenin & & & & & 0.150 & & & \\
\hline Negative & 13 & 3 & 4 & 6 & & & & \\
\hline Low expression & 16 & 4 & 5 & 7 & & & & \\
\hline High expression & 54 & 19 & 20 & 15 & & & & \\
\hline
\end{tabular}

N, Negative; L, low expression; $\mathrm{H}$, high expression. Bold values signify $P$-value $<0.05$.

tumours from patients without a history of tumour recurrence $(P<0.001$ and $<0.001$, respectively). However, there was no significant association between the nuclear and/or cytoplasmic expression of $\beta$-catenin at the tumour invasive front with clinicopathological factors and survival in oral squamous cell carcinoma patients (data not shown).

\section{Correlation between the expression of vimentin,} $E$-cadherin, and $\beta$-catenin

The association between the expression levels of vimentin, E-cadherin, and $\beta$-catenin is shown in Table 3. The expression level of vimentin was inversely associated with E-cadherin $(P=0.035)$, whereas E-cadherin expression was directly associated with $\beta$-catenin $(P=0.01)$. Similarly, there was statistically significant correlation between the expression of vimentin and E-cadherin $(P=0.005)$, and E-cadherin and $\beta$-catenin $(P<0.001)$ at the tumour invasive front (data not shown). However, no correlations were found between the expression of vimentin and $\beta$-catenin $(P=0.15)$ at the tumour invasive front $(P=0.065)$.

\section{Survival Analysis}

At the time of the last follow-up, 40 of 83 (48\%) patients were alive and disease-free, $2(2 \%)$ patients were alive with recurrent disease, and 41 patients $(50 \%)$ had died of tumour recurrence. The overall survival curve for the 83 oral squamous cell carcinomas is shown in Figure 4a. The estimated 1-, 3-, and 5-year disease-free survival was $84 \%$ (95\% CI: $77-92 \%$ ), $68 \%$ (95\% CI: $58-78 \%$ ), and $58 \%$ (95\% CI: $48-69 \%$ ), respectively. Similarly, the overall rate of survival was $84 \%$ (95\% CI: $77-92 \%$ ) at 1 year, $68 \%$ (95\% CI: $58-78 \%$ ) at 3 years, and $58 \%$ (95\% CI: $48-69 \%)$ at 5 years.

In a univariate Cox proportional hazard regression model analysis (Table 4), tumour size $(P=0.044)$, node status $(P=0.049)$, and the expression levels of vimentin $(P<0.001$, Figure $4 b)$, E-cadherin $(P<0.001$
Figure 4c) and $\beta$-catenin $(P=0.01$, Figure $4 d)$ were significantly associated with overall survival. Similarly, tumour size $(P=0.037)$ and the expression levels of vimentin $(P<0.001)$, E-cadherin $(P=0.001)$, and $\beta$-catenin $(P=0.012)$ were significantly associated with disease-free survival (data not shown). Therefore, patients with tumours with negative or low expression of vimentin had a better prognosis than those with tumours with a high vimentin expression level. Conversely, patients with tumours with high E-cadherin or $\beta$-catenin expression had a better prognosis than those with tumours with negative or low expression of E-cadherin or $\beta$-catenin.

In a multivariate Cox regression analysis (Table 5), the expression levels of vimentin $(P=0.042)$ and E-cadherin $(P=0.016)$ showed a significant association with overall survival. Similarly, the expression levels of vimentin $(P=0.032)$ and E-cadherin $(P=0.022)$ showed a significant association with disease-free survival (data not shown). However, a significant correlation between $\beta$-catenin expression and the disease-free survival or overall survival was not shown $(P=0.204)$.

\section{Discussion}

This study quantified the upregulation of vimentin and aberrant expression of E-cadherin/ $\beta$-catenin complexes in biopsies of primary oral squamous cell carcinoma patients using immunohistochemistry. The relationship between the expression levels of these molecular markers and the clinicopathological features of the patients was analysed.

The results of this study show an inverse correlation between vimentin and E-cadherin expression in oral squamous cell carcinoma specimens. Specifically, the overexpression of vimentin was closely associated with the absence or reduced expression of E-cadherin at the invasive front of tumours. E-cadherin, along with catenins, is well recognised for its elective expression and specific roles in epithelial cellular states. ${ }^{27,39}$ Vimentin expression, coupled with the reduced or lack of E-cadherin expression, is characteristic of cells of mesenchymal origin, whereas the converse is true for cells with an epithelial phenotype. ${ }^{27,39}$ These findings support the results of previous in vivo experiments in which vimentin expression resulted in the downregulation of E-cadherin expression. This epithelialmesenchymal transition might have an important role in oral squamous cell carcinoma carcinogenesis or progression. ${ }^{8-10,39,40}$ We also showed a direct correlation between $\beta$-catenin and E-cadherin expression in oral squamous cell carcinoma specimens. However, in this study, vimentin expression failed to correlate with $\beta$-catenin expression, suggesting that either the $\beta$-catenin/TCF pathway may not have a role in the regulation of vimen$\operatorname{tin}^{32,39,41}$ or that there were too few samples to 
a

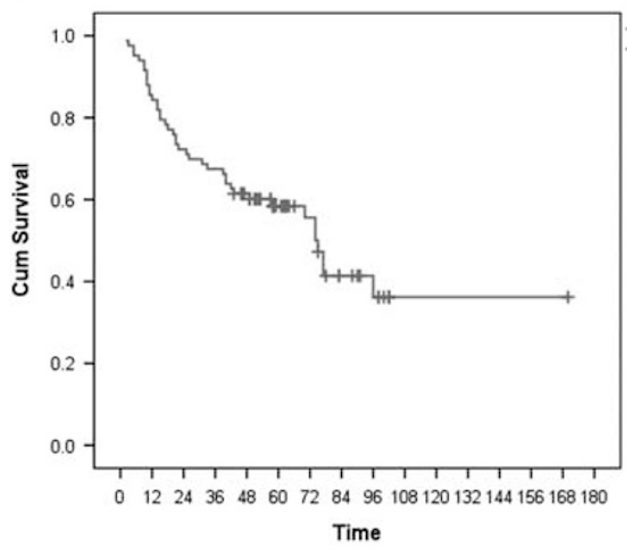

C

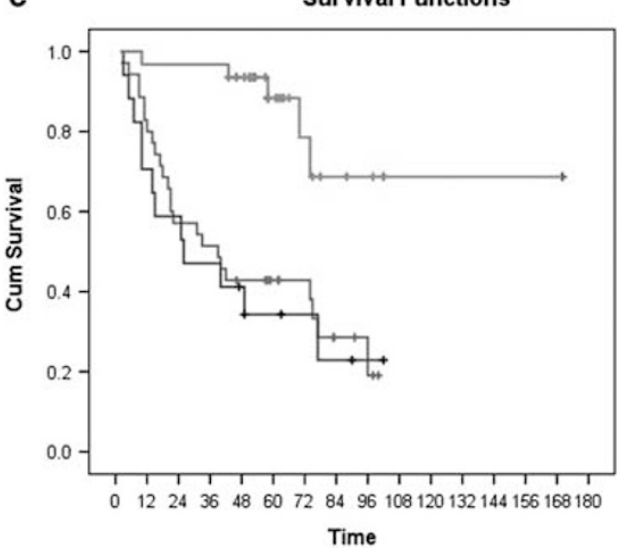

b

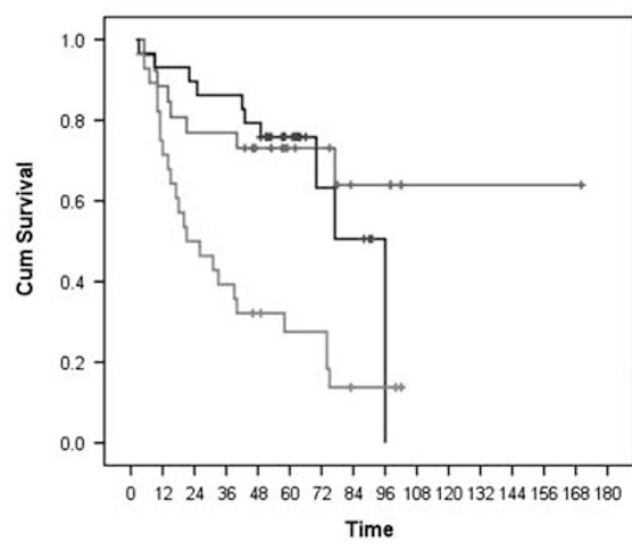

Vimentin

$\neg N$

느

$+\mathrm{N}$-censored

+ L-censored

$+\mathrm{H}$-censored

d

Survival Functions
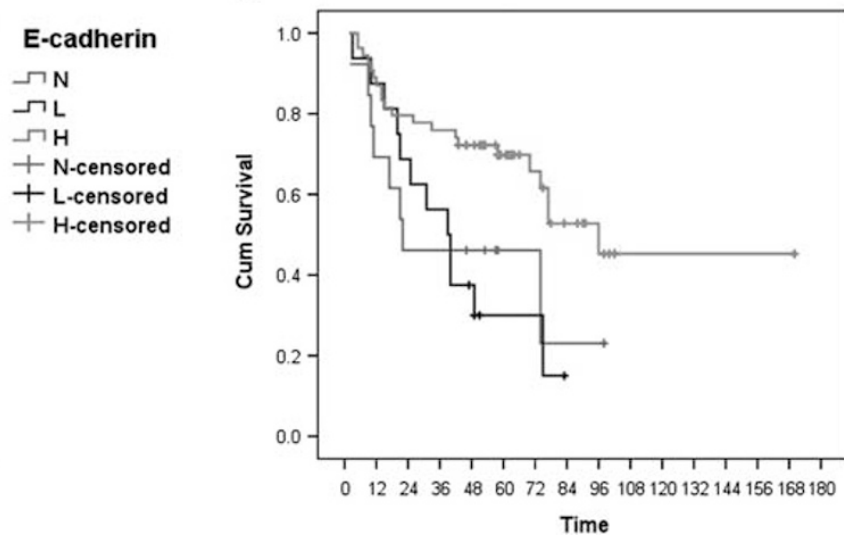

$\beta$-catenin

$\neg N$

$\neg$ L

$+\mathrm{N}$-censored

+ L-censored

+ H-censored

Figure 4 Survival curves of 83 oral squamous cell carcinoma patients. (a) Overall survival curves of 83 oral squamous cell carcinoma patients. (b) Survival curves of 83 oral squamous cell carcinoma patients with tumours lacking or expressing a low or high level of vimentin (log-rank test, $P<0.001$ ). (c) Survival curves of 83 oral squamous cell carcinoma patients with tumours lacking or expressing a low or high level of E-cadherin (log-rank test, $P<0.001$ ). (d) Survival curves of 83 oral squamous cell carcinoma patients with tumours lacking or expressing a low or high level of $\beta$-catenin (log-rank test, $P=0.010$ ). N, negative; L, low expression; $\mathrm{H}$, high expression.

Table 4 Univariate Cox regression analysis of overall survival

\begin{tabular}{|c|c|c|c|}
\hline Covariate & P-value & Risk ratio & $95 \% C I$ \\
\hline Sex (male, female) & 0.603 & 0.848 & $(0.454,1.581)$ \\
\hline Age $(\leq 50,>50$ years $)$ & 0.955 & 0.999 & $(0.972,1.027)$ \\
\hline $\begin{array}{l}\text { Tumor location (tongue, buccal mucosa, gingiva, } \\
\text { palate, lip, floor of the mouth, retromolar pad, others) }\end{array}$ & 0.629 & 0.959 & $(0.810,1.136)$ \\
\hline Histological differentiation (G1, G2, G3) & 0.294 & 1.298 & $(0.798,2.113)$ \\
\hline Pattern of invasion $(1,2,3,4)$ & 0.178 & 1.260 & $(0.900,1.763)$ \\
\hline Tumor size (T1-T4) & 0.044 & 0.739 & $(0.550,0.992)$ \\
\hline Clinical stage (I, II, III, IV) & 0.120 & 0.796 & $(0.598,1.061)$ \\
\hline Nodal metastasis (No, N (+)) & 0.049 & 1.858 & $(1.004,3.440)$ \\
\hline Vimentin expression (negative, low, high) & $<\mathbf{0 . 0 0 1}$ & 2.213 & $(1.445,3.387)$ \\
\hline E-cadherin expression (negative, low, high) & $<0.001$ & 0.502 & $(0.342,0.736)$ \\
\hline$\beta$-Catenin expression (negative, low, high) & 0.010 & 0.615 & $(0.425,0.890)$ \\
\hline
\end{tabular}

No, no nodal metastasis; $\mathrm{N}(+)$, nodal metastasis.

Bold values signify $P$-value $<0.05$.

effectively evaluate the relationship between vimentin expression and the $\beta$-catenin/TCF pathway in tumour cells. Further research is, therefore, necessary to confirm the results of this study.
Many clinicopathological parameters and molecular biomarkers have been associated with local regional recurrence or death in oral squamous cell carcinoma patients. However, clinicopathological 
Table 5 multivariate Cox regression analysis of overall survival

\begin{tabular}{lrrr}
\hline Covariate & P-value & Risk ratio & 95\% CI \\
\hline Sex (male, female) & 0.204 & 0.633 & $(0.312,1.282)$ \\
Age ( $\leq 50,>50$ years) & 0.701 & 0.854 & $(0.382,1.909)$ \\
Tumor location (tongue, buccal mucosa, gingiva, & 0.119 & 0.855 & $(0.702,1.041)$ \\
$\quad$ palate, lip, floor of the mouth, retromolar pad, others) & & 1.307 & $(0.752,2.272)$ \\
Histological differentiation (G1, G2, G3) & 0.343 & 1.000 & $(0.694,1.440)$ \\
Pattern of invasion (1, 2, 3, 4) & 0.998 & 0.395 & $(0.138,1.130)$ \\
Tumor size (T1-T4) & 0.083 & 1.691 & $(0.590,4.851)$ \\
Clinical stage (I, II, III, IV) & 0.328 & 1.711 & $(1.017,2.554)$ \\
Nodal metastasis (N0, N (+)) & 0.139 & 1.612 & $(0.372,0.903)$ \\
Vimentin expression (negative, low, high) & $\mathbf{0 . 0 4 2}$ & 0.579 & $(0.492,1.164)$ \\
E-cadherin expression (negative, low, high) & $\mathbf{0 . 0 1 6}$ & 0.757 & \\
-Catenin expression (negative, low, high) & 0.204 & &
\end{tabular}

No, no nodal metastasis; $\mathrm{N}(+)$, nodal metastasis.

Bold values signify $P$-value $<0.05$.

variables and tumour-specific molecular markers that can identify patients with the highest risk of local recurrence have yet to be defined. ${ }^{16,18-23,42-49}$

Vimentin, a mesenchymal cell marker, associates with components of the cytoskeleton and membrane adhesions. Studies of human epithelial carcinomas, such as breast cancer, hepatocellular carcinoma, colon carcinoma, and prostatic adenocarcinoma, have shown that vimentin expression can be correlated with tumour invasion and a poor prognosis. ${ }^{8-11,27-32}$ Several previous publications have detected the expression of vimentin in oral squamous cell carcinoma patients or cell lines. ${ }^{8,40,50}$ To our knowledge, this is the first study to show that the overexpression of vimentin in oral squamous cell carcinoma specimens was closely associated with local recurrence. No other clinicopathological factors were significantly associated with vimentin expression. Using univariate or multivariate analyses, the overexpression of vimentin showed a significant association with a short overall or disease-free survival in oral squamous cell carcinoma patients. In oral squamous cell carcinoma patients, tumours lacking or expressing a low level of vimentin were correlated with a better prognosis than tumours with high vimentin expression. Moreover, strong vimentin expression was found in the invading tumour cells at the invasive front. On the basis of these findings, this study suggests that vimentin may provide prognostically relevant information on the biological behaviour of oral squamous cell carcinoma.

The clinicopathological significance of E-cadherin and $\beta$-catenin expression in whole-tumour sections from oral squamous cell carcinoma patients is controversial. The absence or low expression of E-cadherin or $\beta$-catenin was significantly associated with some clinicopathological parameters and with a poor prognosis in some publications, but not in others. ${ }^{19-23,51-54}$ The reason for these contradictory results may be that the standard used to evaluate the immunostaining and the definition of reduced or overexpression is quite variable. Alternatively, the tumour cells in oral squamous cell carcinoma patients may exhibit a heterogeneous cell population with variable behaviour. ${ }^{19,51}$ Some investigators have reported that the lack of or reduced E-cadherin expression in oral squamous cell carcinoma was correlated with several clinicopathological factors, such as a high tumour grade, a lower degree of differentiation, and regional lymph node metastasis. ${ }^{20-22,27,51}$ However, in this study, the expression level of E-cadherin or $\beta$-catenin was not significantly associated with sex, age, tumour location, histological differentiation, pattern of invasion, tumour size, clinical stage, or nodal metastasis. However, the absence of or reduced E-cadherin expression was significantly associated with local recurrence and a short overall or disease-free survival in oral squamous cell carcinoma patients. Conversely, the high expression of E-cadherin in tumours was correlated with a better prognosis than the absence of or reduced E-cadherin expression in tumours. This may indicate that E-cadherin expression is an independent prognostic factor in univariate or multivariate analysis. ${ }^{18,20,21,55}$

In contrast to E-cadherin expression, we found that the absence of or low $\beta$-catenin expression in sections of whole tumour was not significantly associated with local recurrence or with a short survival. However, our results showed that the absence of or low $\beta$-catenin expression at the tumour invasive front associated with local recurrence and with a short survival. Pukkila et $a l^{56}$ observed a correlation between the nuclear expression of $\beta$-catenin and patient survival, whereas in our study, no such correlation was found. ${ }^{57}$ Therefore, there is a need for further studies on $\beta$-catenin in oral carcinomas. Although $\beta$-catenin expression at the tumour invasive front was a prognostic factor in the univariate analysis, it was not an independent factor in the multivariate analysis in this study. Therefore, our data suggest that $\beta$-catenin expression at the tumour invasive front may function as a clinically relevant tumour marker in conjunction with other clinicopathological variables in the prognosis of oral squamous cell carcinoma patients. To date, the results from studies examining the correlation 
between E-cadherin and $\beta$-catenin expression and the prognosis of oral squamous cell carcinoma patients have been controversial. ${ }^{19-23,51-54}$ Therefore, further studies with a large patient population are needed to examine the role of E-cadherin and $\beta$-catenin using standardised methods to evaluate the immunostaining and with standardised definitions of reduced or overexpression.

In conclusion, these data show that the overexpression of vimentin was closely associated with local recurrence and survival in oral squamous cell carcinoma patients. The combination of the upregulation of vimentin and aberrant expression of E-cadherin/ $\beta$-catenin complexes at the tumour invasive front in oral squamous cell carcinoma patients may provide useful, prognostically relevant data on the biological behaviour of oral squamous cell carcinoma.

\section{Acknowledgements}

We thank Dr Chanjuan Li for assistance with statistical data presentation. We also thank Sheng Chen and Wei Zhang for excellent technical assistance. This study was supported by grant from the National Natural Science Foundation of China (Grant no. 30872903) and from the Key Project of Health Department of Jiangsu Province (Grant no. H200753), and from Project of Science and Technology Department of Jiangsu Province (Grant no. BK2008362). We thank American Journal Experts for revising the English used in this article.

\section{Disclosure/conflict of interest}

The authors declare no conflict of interest.

\section{References}

1 Forastiere AA, Ang K, Brizel D, et al. Head and neck cancers. J Natl Compr Canc Netw 2005;3:316-391.

2 Hunter KD, Parkinson EK, Harrison PR. Profiling early head and neck cancer. Nat Rev Cancer 2005;5:127-135.

3 Casiglia J, Woo SB. A comprehensive review of oral cancer. Gen Dent 2001;49:72-82.

4 Vokes EE, Weichselbaum RR, Lippman SM, et al. Head and neck cancer. N Engl J Med 1993;328:184-194.

5 Bettendorf O, Piffkò J, Bànkfalvi A. Prognostic and predictive factors in oral squamous cell cancer: important tools for planning individual therapy? Oral Oncol 2004;40:110-119.

6 Bryne M, Koppang HS, Lilleng R, et al. Malignancy grading of the deep invasive margins of oral squamous cell carcinomas has high prognostic value. J Pathol 1992;166:375-381.

7 Woolgar JA. Histopathological prognosticators in oral and oropharyngeal squamous cell carcinoma. Oral Oncol 2006;42:229-239.

8 Mandal M, Myers JN, Lippman SM, et al. Epithelial to mesenchymal transition in head and neck squamous carcinoma: association of Src activation with E-cadherin down-regulation, vimentin expression, and aggressive tumor features. Cancer 2008;112:2088-2100.

9 Huber MA, Kraut N, Beug H. Molecular requirements for epithelial-mesenchymal transition during tumor progression. Curr Opin Cell Biol 2005;17:548-558.

10 Christiansen JJ, Rajasekaran AK. Reassessing epithelial to mesenchymal transition as a prerequisite for carcinoma invasion and metastasis. Cancer Res 2006;66:8319-8326.

11 Thiery JP. Epithelial-mesenchymal transitions in development and pathologies. Curr Opin Cell Biol 2003;15:740-746.

12 Nawshad A, LaGamba D, Polad A, et al. Transforming growth factor- $\beta$ Signaling during epithelial-mesenchymal transformation: implications for embryogenesis and tumor metastasis. Cells Tissues Organs 2005;179:11-23.

13 Behrens J. Cadherins and catenins: role in signal transduction and tumor progression. Cancer Metastasis Rev 1999;18:15-30.

14 Hsu YM, Chen YF, Chou ChY, et al. KCl cotransporter-3 down-regulates E-cadherin/ $\beta$-catenin complex to promote epithelial-mesenchymal transition. Cancer Res 2007;67:11064-11073.

15 Zheng Z, Pan J, Chu B, et al. Downregulation and abnormal expression of E-cadherin and $\beta$-catenin in nasopharyngeal carcinoma: close association with advanced disease stage and lymph node metastasis. Hum Pathol 1999;30:458-466.

16 Pyo SW, Hashimoto M, Kim YS, et al. Expression of E-cadherin, P-cadherin and $\mathrm{N}$-cadherin in oral squamous cell carcinoma: correlation with the clinicopathologic features and patient outcome. J Craniomaxillofac Surg 2007;35:1-9.

17 Chang HW, Chow V, Lam KY, et al. Loss of E-cadherin expression resulting from promoter hypermethylation in oral tongue carcinoma and its prognostic significance. Cancer 2002;94:386-392.

18 Diniz-Freitas M, García-Caballero T, Antúnez-López J, et al. Reduced E-cadherin expression is an indicator of unfavourable prognosis in oral squamous cell carcinoma. Oral Oncol 2006;42:190-200.

19 Mahomed F, Altini M, Meer S. Altered E-cadherin/ $\beta$-catenin expression in oral squamous carcinoma with and without nodal metastasis. Oral Dis 2007;13: 386-392.

20 Tanaka N, Odajima T, Ogi K, et al. Expression of E-caderin, $\alpha$-catenine, and $\beta$-cetenin in the process of lymph node metastasis in oral squamous cell carcinoma. Br J Cancer 2003;89:557-563.

21 Andrews NA, Jones AS, Helliwell TR, et al. Expression of the E-cadherin-catenin cell adhesion complex in primary squamous cell carcinomas of the head and neck and their nodal metastases. $\mathrm{Br} \mathrm{J}$ Cancer 1997;75:1474-1480.

22 Chow V, Yuen AP, Lam KY, et al. A comparative study of the clinicopathological significance of E-cadherin and catenins $(\alpha, \beta, \gamma)$ expression in the surgical management of oral tongue carcinomas. J Cancer Res Clin Oncol 2001;127:59-63.

23 Bánkfalvi A, Krassort M, Végh A, et al. Deranged expression of the E-cadherin/beta-catenin complex and the epidermal growth factor receptor in the clinical evolution and progression of oral squamous cell carcinomas. J Oral Pathol Med 2002;31:450-457.

24 Takeichi M. Cadherin cell adhesion receptors as a morphogenetic regulator. Science 1991;251:1451-1455. 
25 Behrens J, von Kries JP, Kühl M, et al. Functional interaction of $\beta$-catenin with the transcription factor LEF-1. Nature 1996;382:638-642.

26 Conacci-Sorrell M, Zhurinsky J, Ben Ze'ev A. The cadherin-catenin adhesion system in signaling and cancer. J Clin Invest 2002;109:987-991.

27 Steinert PM, Roop DR. Molecular and cellular biology of intermediate filaments. Annu Rev Biochem 1988;57:593-625.

28 Ramaekers FC, Haag D, Kant A, et al. Coexpression of keratin- and vimentin-type intermediate filaments in human metastatic carcinoma cells. Proc Natl Acad Sci USA 1983;80:2618-2622.

29 Gilles C, Polette M, Piette J, et al. Vimentin expression in cervical carcinomas: association with invasive and migratory potential. J Pathol 1996;180:175-180.

30 Gilles C, Polette M, Zahm JM, et al. Vimentin contributes to human mammary epithelial cell migration. J Cell Sci 1999;112:4615-4625.

31 Paccione RJ, Miyazaki H, Patel V, et al. Keratin downregulation in vimentin-positive cancer cells is reversible by vimentin RNA interference, which inhibits growth and motility. Mol Cancer Ther 2008;7:28942903.

32 Gilles C, Polette M, Mestdagt Me, et al. Transactivation of vimentin by $\beta$-catenin in human breast cancer cells. Cancer Res 2003;63:2658-2664.

33 Bryne M, Boysen M, Alfsen CG, et al. The invasive front of carcinomas. The most important area for tumor prognosis? Anticancer Res 1998;18:4757-4764.

34 Bànkfalvi A, Piffkò J. Prognostic and predictive factors in oral cancer: the role of the invasive tumor front. J Oral Pathol Med 2000;29:291-298.

35 Bryne M, Koppang HS, Lilleng R, et al. New malignancy grading is a better prognostic indicator than Broders' grading in oral squamous cell carcinomas. J Oral Pathol Med 1989;18:432-437.

36 Bryne $M$, Jenssen N, Boysen M. Histological grading in the deep invasive front of $\mathrm{T} 1$ and $\mathrm{T} 2$ glottic squamous cell carcinomas has high prognostic value. Virchows Arch 1995;427:277-281.

37 Chui X, Egami H, Yamashita J, et al. Immunohistochemical expression of the c-kit proto-oncogene product in human malignant and non-malignant breast tissues. Br J Cancer 1996;73:1233-1236.

38 Engels K, Knauer SK, Metzler D, et al. Dynamic intracellular survivin in oral squamous cell carcinoma: underlying molecular mechanism and potential as an early prognostic marker. J Pathol 2007;211:532-540.

39 Kokkinos MI, Wafai R, Wong MK, et al. Vimentin and epithelial-mesenchymal transition in human breast cancer-observations in vitro and in vivo. Cells Tissues Organs 2007;185:191-203.

40 Islam S, Kim JB, Trendel J, et al. Vimentin expression in human squamous carcinoma cells: relationship with phenotypic changes and cadherin-based cell adhesion. J Cell Biochem 2000;78:141-150.

41 Bindels S, Mestdagt M, Vandewalle C, et al. Regulation of vimentin by SIP1 in human epithelial breast tumor cells. Oncogene 2006;25:4975-4985.

42 Sasahira T, Kirita T, Bhawal UK, et al. Receptor for advanced glycation end products (RAGE) is important in the prediction of recurrence in human oral squamous cell carcinoma. Histopathology 2007;51:166-172.

43 Keski-Säntti H, Atula T, Tikka J, et al. Predictive value of histopathologic parameters in early squamous cell carcinoma of oral tongue. Oral Oncol 2007;43:10071013.

44 de Vicente1 JC, Lequerica-Fernández P, Santamaría J, et al. Expression of MMP-7 and MT1-MMP in oral squamous cell carcinoma as predictive indicator for tumor invasion and prognosis. J Oral Pathol Med 2007;36:415-424.

45 Lin PY, Yu ChH, Wang JT, et al. Expression of hypoxiainducible factor-1a is significantly associated with the progression and prognosis of oral squamous cell carcinomas in Taiwan. J Oral Pathol Med 2008;37:1825.

46 Yanamoto S, Kawasaki G, Yoshitomi I, et al. Clinicopathologic significance of EpCAM expression in squamous cell carcinoma of the tongue and its possibility as a potential target for tongue cancer gene therapy. Oral Oncol 2007;43:869-877.

47 Brandwein-Gensler M, Teixeira MS, Lewis CM, et al. Oral squamous cell carcinoma histologic risk assessment, but not margin status, is strongly predictive of local disease-free and overall survival. Am J Surg Pathol 2005;29:167-178.

48 de Aguiar Jr AF, Kowalski LP, de Almeida OP. Clinicopathological and immunohistochemical evaluation of oral squamous cell carcinoma in patients with early local recurrence. Oral Oncol 2007;43:593601.

49 Ginos MA, Page GP, Michalowicz BS, et al. Identification of a gene expression signature associate with recurrent disease in squamous cell carcinoma of head and neck. Cancer Res 2004;64:55-63.

50 de Araujo VC, Pinto Júnior DS, de Sousa SO, et al. Vimentin in oral squamous cell carcinoma. Eur Arch Otorhinolaryngol 1993;250:105-109.

51 Wang X, Zhang J, Fan M, et al. The expression of E-cadherin at the invasive tumor front of oral squamous cell carcinoma: immunohistochemical and RT-PCR analysis with clinicopathological correlation. Oral Surg Oral Med Oral Pathol Oral Radiol Endod 2009;107:547-554.

52 Bánkfalvi A, Krassort M, Buchwalow IB, et al. Gains and losses of adhesion molecules (CD44, E-cadherin, and beta-catenin) during oral carcinogenesis and tumour progression. J Pathol 2002;198:343-351.

53 Ueda G, Sunakawa H, Nakamori K, et al. Aberrant expression of beta and gamma-catenin is an independent prognostic marker in oral squamous cell carcinoma. Int J Oral Maxillofac Surg 2006;35:356-361.

54 Foschini MP, Cocchi R, Morandi L, et al. E-Cadherin loss and DNP73L expression in oral squamous cell carcinomas showing aggressive behavior. Head Neck 2008;30:1475-1482.

55 Kurtz KA, Hoffman HT, Zimmerman MB, et al. Decreased E-cadherin but not $\beta$-catenin expressionis associated with vascular invasion and decreased survival in head and neck squamous carcinomas. Otolaryngol Head Neck Surg 2006;134:142-146.

56 Pukkila MJ, Virtaniem JA, Kumpulainen EJ, et al. Nuclear $\beta$-catenin is related to unfavourable outcome in oropharyngeal and hypopharyngeal squamous cell carcinoma. J Clin Pathol 2001;54:42-47.

57 de Castro J, Gamallo C, Palacios J, et al. $\beta$-Catenin expression pattern in primary esophageal squamous cell carcinoma. Relationship with clinicopathologic features and clinical outcome. Virchows Arch 2000;437:599-604. 